\title{
DESUBLIMATION ON THE SURFACE OF AN AN- NULAR-TUBE COLD TRAP TO BE OPERATED IN A TRANSITION REGION WITH A CLOSED SYSTEM
}

\author{
TETSUIRO MITANI AND YoICHI TAKASHIMA \\ Research Laboratory of Nuclear Reactors, \\ Tokyo Institute of Technology, Tokyo 152
}

\begin{abstract}
To have some phenomenological and fundamental information on the desublimation processes in an annular-tube cold trap to be operated with a closed system at such low pressures that the Navier-Stokes equations may not be valid, we made experimental studies on the desublimation processes of naphthalene vapor in stationary nitrogen gas.

It was found that the desublimation process consisted of an initial transient period and a subsequent quasi-steady period. Because the latter should be important for the design of the cold trap, under the quasi-steady period, we estimated three characteristic quantities such as the local desublimation rate, the apparent density and the reference length of desublimated solid layer. The effects of operating conditions on these quantities were studied by means of new dimensionless parameters, $M a$ and $P e$ which were induced from our theoretical development based on Maxwell's moment method. It was found that the desublimation process in the transition region depended strongly upon $\mathrm{Ma}$ but scarcely upon $P e$.
\end{abstract}

\section{Introduction}

A number of cold traps have been used for various purposes in many chemical processes. Some of them, e.g., those used in a molecular distillation process or in a freeze drying process, are operated at such a low pressure that the Navier-Stokes equations may not be valid, for the purpose of collecting vapor from air which has slightly leaked on the way. In most cases the leaking rate is negligibly small in comparison with the vapor feed rate. Therefore, it is natural as well as desirable to operate with a closed system for high collection efficiency, especially in trapping a valuable vapor such as $U F_{6}$. But a small amount of the vapor mixture must be purged with air at a moderate interval if the total pressure in the cold trap is to be kept low.

We may consider the above desublimation process as a process in which vapor molecules transfer through stationary noncondensable gas and desublimate on the cryosurface of the cold trap. If in the continuum region, the dominant resistance when vapor molecules transfer and desublimate is a diffusional one due to molecule-molecule collisions. On the other hand, in the free molecular flow region, the resistance of changing phase due to desublimation and the resist-

Received July 18, 1980. Correspondence concerning this atticle should be addressed to T. Mitani. ance of flow due to molecule-wall collisions are dominant. Therefore, it should be valuable to study the desublimation processes in such a cold trap as being operated with a closed system over various rarefactions from the continuum region to the free molecular flow region.

As for investigation of a cold trap or a desublimation process, in the continuum region, fundamental and phenomenological studies of various desublimations in forced or free convection have been made by many investigators ${ }^{1,2,4-\hat{6}, 9,17-20)}$, in which some useful information on crystal form, thermal conductivity or densification of desublimated solid phase are reported. Also there have been some studies ${ }^{4,5,8,17)}$ aimed at a proper method of design. However, in the transition region or in the free molecular flow region, there have been a few fundamental studies ${ }^{3,10,14-16)}$ on desublimation but none on design nor on the desublimation process.

In the present paper, experimental studies on the desublimation process of naphthalene vapor in stationary nitrogen gas in an annular-tube cold trap are discussed for the transition region, where the Knudsen number is less than about 1.1. The freezing states are directly observed through the transparent outer tube of the cold trap, and the characteristic quantities such as local desublimation rate, apparent density and reference length of desublimated solid layer are 


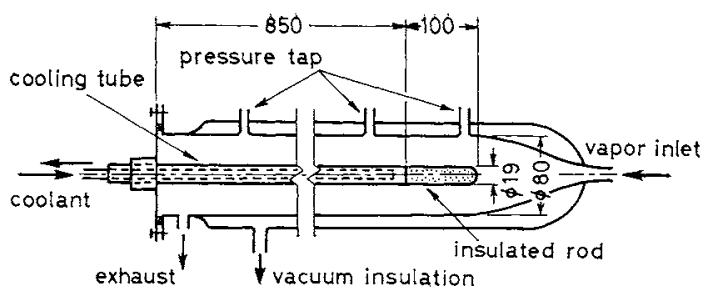

Fig. 1 Details of cold trap

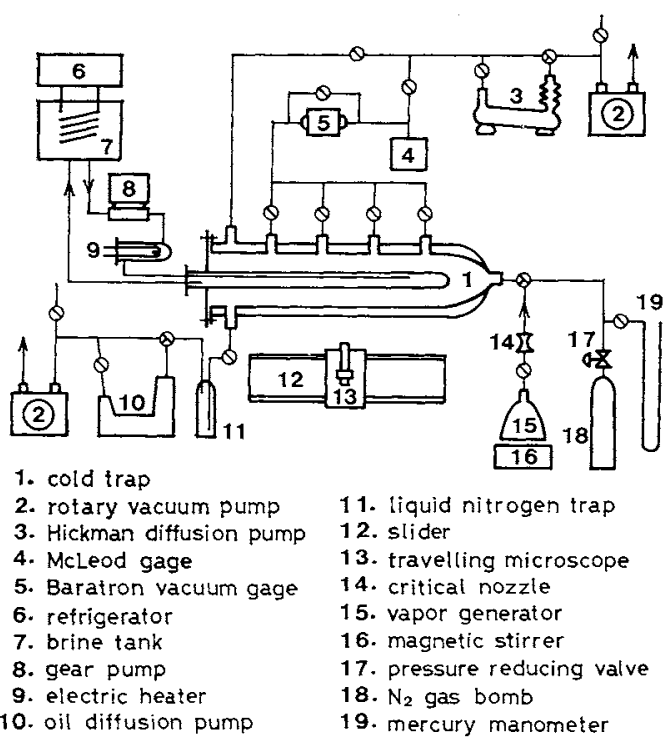

Fig. 2 Flow diagram of experimental apparatus

determined from data on the growing rates of desublimated solid layer. The effects of operating conditions on these quantities are discussed by means of new dimensionless parameters induced from our theoretical development of Maxwell's moment equations $^{11}$, though the details of this theoretical approach are not reported yet.

\section{Experimental Procedure}

The details of the cold trap and the flow diagram of the experimental apparatus are illustrated in Figs. 1 and 2. The inner cooling tube, $19 \mathrm{~mm}$ in diameter, is made of copper and plated nickel. The glass outer tube is $80 \mathrm{~mm}$ in inner diameter and has four pressure taps at distances of 7, 17 and $37 \mathrm{~cm}$ in the axial direction from the border between the insulated rod and the inner cooling tube, and at the front of the insulated rod made of cork. The total pressure in the cold trap was measured by a Baratron vacuum gage. The temperatures at the inner surface of the outer tube and at the inner cooling tube surface were measured by copper-constantan thermocouples $0.1 \mathrm{~mm}$ in diameter fitted at several points along the axial direction. The temperature of the inner cooling tube was controlled by an electric heater installed along the brine pipeline (trichlorethylene). The feed rate of vapor was adjusted by controlling the vapor pressure upstream of a critical nozzle.

Experimental procedure was as follows: (1) Keeping the temperature of the inner cooling tube surface constant by circulating brine. (2) Closing the stop cock leading to the exhaust after adjusting the pressure of nitrogen gas in the cold trap. (3) Feeding pure naphthalene vapor at a constant rate. The temperature of naphthalene vapor drops from about $373 \mathrm{~K}$ upstream of a critical nozzle to room temperature at the entrance of the cold trap because the vapor expands quickly after passing through the critical nozzle. (4) Measuring thickness of desublimated solid layer at each of 5 to 8 points along the axial direction with an accuracy of $0.05 \mathrm{~mm}$ by a travelling microscope at time intervals of 15 to 30 minutes, at the same time measuring the length of desublimated solid layer. (5) Observing the freezing state and taking photograph. (6) Measuring the total feed of vapor at the end of each run by weighing it as collected in a liquid nitrogen trap.

The experimental conditions for all the runs are shown in Table 1. The temperature of the inner cooling tube $T_{c}$ was set at about 225,245 or $263 \mathrm{~K}$. The total pressure in the cold trap $P_{t}$ was varied over a range from 0.14 to $4.7 \mathrm{~Pa}$. The feed rate of vapor $G$ was from 1.2 to $2.0 \mathrm{~g} / \mathrm{hr}$ and the time of each run $\theta$ was from 120 to 240 minutes.

\section{Results and Discussion}

We have observed the following facts. The total pressure is kept constant and almost the same at each pressure tap in the same experiment. The temperature at the inner cooling tube surface is kept constant. The temperatures at the outer tube surface fall so slowly that the effect of their variation on desublimation rate may be negligible. The length of desublimated solid layer is kept nearly constant as shown in Fig. 3. The longest one is about $28 \mathrm{~cm}$, much less than the $85-\mathrm{cm}$ length of the inner cooling tube. The local growing rate of desublimated solid layer at each point becomes slower with time during an early period but is nearly constant after about 45 to 60 minutes, as an example shows in Fig. 4. This tendency is consistent with previous studies ${ }^{2,6,20)}$ of frost deposition that in most cases heat and mass transfer rates become constant after an initial transient period.

From these observations, it is considered that in the present case a quasi-steady state is established after an initial transient period, not only because the thickness of desublimated solid layer, $\delta$, is negligibly small in comparison with the interval between the inner and the outer tube, $\Delta r$, but also the temperature of the desublimation surface tends to remain constant due to an increase in density of desublimated solid 


\begin{tabular}{|c|c|c|c|c|c|c|c|c|c|c|}
\hline \multirow[b]{2}{*}{ Run } & \multirow[b]{2}{*}{$\begin{array}{c}T_{c} \\
{[\mathrm{~K}]}\end{array}$} & \multirow[b]{2}{*}{$\begin{array}{l}T_{\mathrm{ar}} \\
{[\mathrm{K}]}\end{array}$} & \multirow{2}{*}{$\begin{array}{c}\text { Table } 1 \\
\theta \\
{[\mathrm{min}]}\end{array}$} & \multicolumn{4}{|c|}{ Experimental conditions and reference length $Z d^{*}$} & \multirow[b]{2}{*}{$\alpha=80 \%$} & \multirow[b]{2}{*}{$\begin{array}{c}Z d^{*} \\
\alpha=90 \%\end{array}$} & \multirow[b]{2}{*}{$\alpha=95 \%$} \\
\hline & & & & $\begin{array}{c}P_{t} \\
{[\mathrm{~Pa}]}\end{array}$ & $\begin{array}{c}G \\
{[\mathrm{~g} / \mathrm{hr}]}\end{array}$ & $\begin{array}{c}P e \\
{[-]}\end{array}$ & $\begin{array}{l}M a \\
{[-]}\end{array}$ & & & \\
\hline 1 & 224 & 265 & 180 & 0.399 & 1.63 & 0.275 & 0.0596 & 3.10 & 4.54 & 5.62 \\
\hline 2 & 224 & 264 & 225 & 0.180 & 1.67 & 0.283 & 0.136 & 2.77 & 4.43 & 5.72 \\
\hline 3 & 227 & 266 & 120 & 29.46 & 1.95 & 0.326 & 0.00096 & 1.24 & 1.95 & 2.60 \\
\hline 4 & 225 & 263 & 240 & 0.369 & 1.40 & 0.239 & 0.0556 & 2.35 & 3.65 & 4.75 \\
\hline 5 & 225 & 263 & 240 & 0.399 & 1.19 & 0.203 & 0.0437 & 2.52 & 3.85 & 4.88 \\
\hline 6 & 225 & 263 & 180 & 1.947 & 1.32 & 0.226 & 0.00992 & 1.90 & 2.89 & 3.59 \\
\hline 7 & 225 & 263 & 180 & 1.404 & 1.32 & 0.226 & 0.0138 & 1.75 & 2.64 & 3.36 \\
\hline 8 & 224 & 262 & 240 & 0.147 & 1.25 & 0.215 & 0.125 & 2.94 & 4.18 & 5.26 \\
\hline 9 & 225 & 262 & 180 & 0.883 & 1.44 & 0.248 & 0.0239 & 1.51 & 2.26 & 2.99 \\
\hline 10 & 224 & 262 & 150 & 2.720 & 2.01 & 0.346 & 0.0108 & 1.57 & 2.54 & 3.21 \\
\hline 11 & 246 & 269 & 140 & 3.306 & 1.65 & 0.270 & 0.00727 & 1.58 & 2.21 & 2.77 \\
\hline 12 & 245 & 268 & 110 & 3.106 & 1.78 & 0.293 & 0.00836 & 1.35 & 1.93 & 2.54 \\
\hline 13 & 245 & 268 & 180 & 0.283 & 1.87 & 0.308 & 0.103 & 2.91 & 4.22 & 5.48 \\
\hline 14 & 245 & 267 & 150 & 2.520 & 1.70 & 0.282 & 0.00987 & 1.63 & 2.36 & 2.97 \\
\hline 15 & 245 & 268 & 240 & 0.324 & 1.47 & 0.242 & 0.0699 & 3.22 & 4.57 & 5.62 \\
\hline 16 & 245 & 268 & 225 & 1.507 & 1.54 & 0.254 & 0.0150 & 2.63 & 3.98 & 5.08 \\
\hline 17 & 245 & 268 & 210 & 3.400 & 1.83 & 0.301 & 0.00784 & 2.11 & 2.94 & 3.51 \\
\hline 18 & 242 & 266 & 240 & 0.398 & 1.65 & 0.276 & 0.0622 & 3.46 & 4.57 & 5.43 \\
\hline 19 & 245 & 268 & 150 & 2.040 & 2.85 & 0.469 & 0.0204 & 1.71 & 2.44 & 3.05 \\
\hline 20 & 264 & 279 & 165 & 2.613 & 1.67 & 0.254 & 0.0100 & 1.88 & 2.52 & 3.05 \\
\hline 21 & 264 & 279 & 150 & 4.733 & 1.81 & 0.275 & 0.00574 & 1.77 & 2.44 & 2.97 \\
\hline 22 & 264 & 278 & 180 & 0.476 & 1.65 & 0.253 & 0.105 & 3.64 & 4.77 & 5.62 \\
\hline 23 & 263 & 279 & 240 & 1.773 & 1.79 & 0.272 & 0.0164 & 1.99 & 2.79 & 3.37 \\
\hline 24 & 260 & 278 & 180 & 0.180 & 1.57 & 0.240 & 0.660 & 4.77 & 6.01 & 6.90 \\
\hline 25 & 263 & 278 & 210 & 0.551 & 1.85 & 0.283 & 0.0800 & 3.55 & 4.51 & 5.21 \\
\hline 26 & 260 & 278 & 185 & 0.195 & 1.46 & 0.223 & 0.474 & 4.70 & 5.91 & 6.78 \\
\hline 27 & 263 & 278 & 210 & 4.013 & 1.95 & 0.298 & 0.00732 & 1.85 & 2.52 & 3.16 \\
\hline 28 & 263 & 278 & 240 & 2.906 & 1.75 & 0.268 & 0.00928 & 1.77 & 2.42 & 2.93 \\
\hline
\end{tabular}

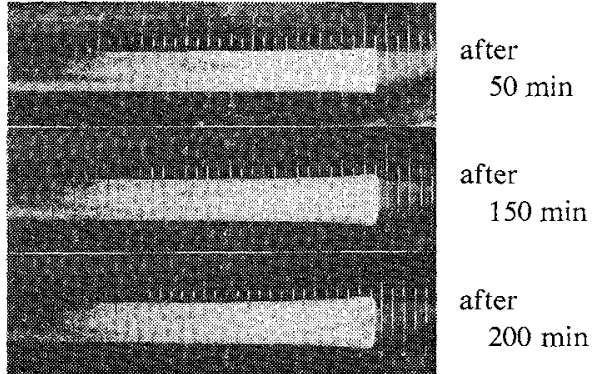

Fig. 3 Photographs at several elapsed time for Run 28

phase $^{2,20)}$.

Under such quasi-steady states as mentioned above, we have estimated three characteristic quantities for desublimation (local desublimation rate, apparent density and reference length of desublimated solid layer) from the data of the volumetric increasing rates obtained by integrating numerically the profiles of the solid layer as shown in Fig. 5. The plotted points are interpolated in Fig. 4.

We have studied the effects of experimental conditions on these characteristic quantities by means of $M a$ and $P e$ since the transport behavior should depend on $M a, P e$ and accommodation coefficient, according to our theoretical development based on Maxwell's moment method ${ }^{11)}$, though the details of this theoretical approach are not reported yet. $M a$ and $P e$ are new dimensionless parameters obtained

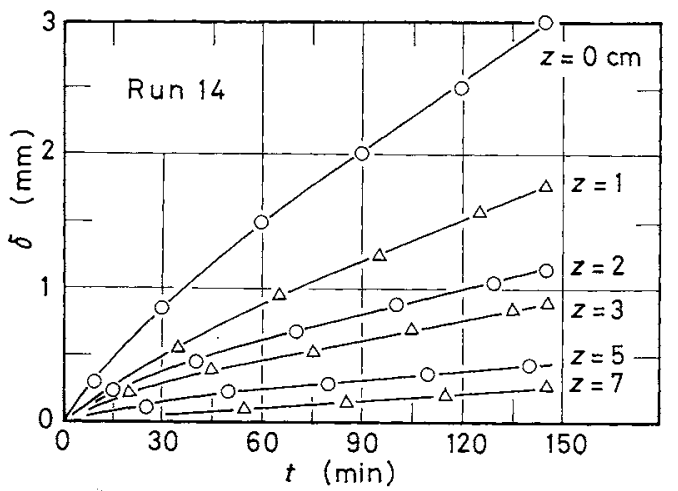

Fig. 4 Time variation of desublimated solid layer thickness

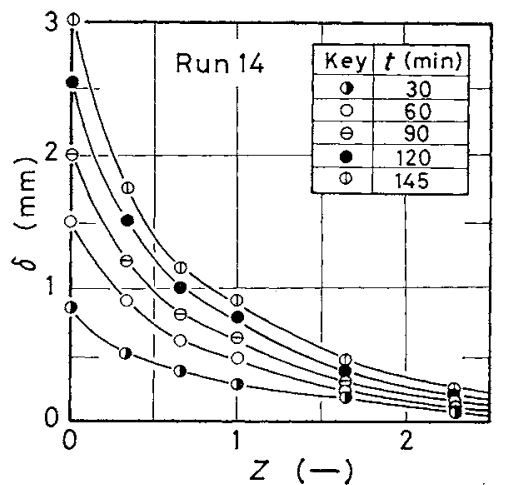

Fig. 5 Time variation of desublimated solid layer profile 


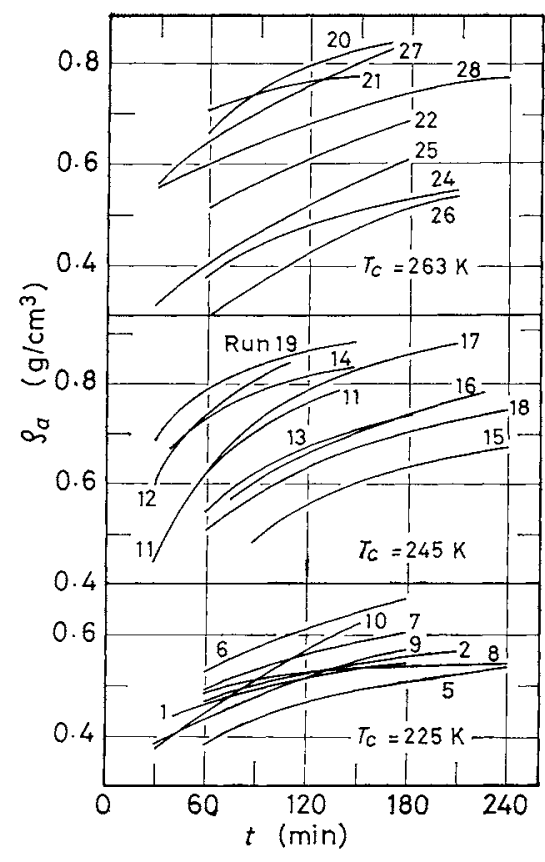

Fig. 6 Time dependence of apparent density

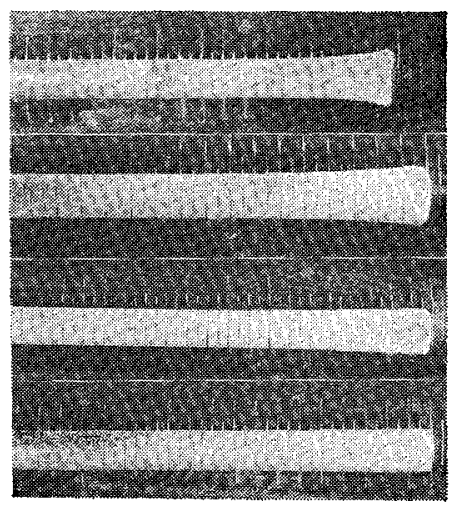

Run 3

$T_{\mathrm{c}}=227 \mathrm{~K}$

$P_{t}=29.46 \mathrm{~Pa}$

Run 9

$T_{c}=225 \mathrm{~K}$

$P_{t}=0.883 \mathrm{~Pa}$

Run 8

$T_{c}=224 \mathrm{~K}$

$P_{t}=0.147 \mathrm{~Pa}$

Run 22

$T_{c}=264 \mathrm{~K}$

$P_{t}=0.476 \mathrm{~Pa}$

Fig. 7 Photographs at the end of experiment

by combining the moment equations with the boundary and the operating conditions.

Assuming that a binary diffusivity $D_{A B}$ is represented in a general formula such as

$$
D_{A B}=D_{o}\left(\frac{T_{\mathrm{QV}}}{273.15}\right)^{n}\left(\frac{101300}{P_{t}}\right)
$$

$M a$ and $P e$ are defined in the SI units system as follows:

$$
\begin{aligned}
& M a=\left(\frac{11207}{\pi r_{1} \Delta r M_{A} \beta_{A}}\right)\left(\frac{101300}{P_{t}-P_{A S}}\right) G \\
& P e=\left(\frac{11207}{\pi r_{1} M_{A} D_{0}}\right)\left(\frac{273.15}{T_{\mathrm{av}}}\right)^{n} G
\end{aligned}
$$

Where $M_{A}$ is the molecular weight of vapor and $D_{o}$ is the binary diffusivity at the normal condition. $\beta_{A}$ is the most probable velocity of vapor and $P_{A S}$ is the saturated vapor pressure at $T_{c}$. $n$ is derived to be 1.5 from the classical kinetic theory ${ }^{7}$, but strictly it is variable from 1.5 to 2.0 with the intermolecular force.
$T_{\mathrm{av}}$ is the arithmetical average between the outer and the inner tube surface temperature. Equations (2) and (3) show that $M a$ is directly proportional to $G$ and inversely to the difference between $P_{t}$ and $P_{A S}$ and $P e$ is proportional to $G$.

The values of $M a$ and $P e$ for all the runs are shown in Table 1. The values of $M a$ were obtained by estimating the $P_{A S}$ from the following empirical formula ${ }^{8}$.

$$
\ln \left(P_{A S}\right)=32.0166-\left(8778.24 / T_{c}\right)-0.02583 \ln \left(T_{c}\right)
$$

The values of $P e$ were calculated by assuming $n=2.0$ and $D_{o}=0.0513 \mathrm{~cm}^{2} / \mathrm{s}$ which are for the mixture of air and naphthalene ${ }^{13)}$.

The accommodation coefficient of naphthalene scarcely depends on the temperature of the desublimation surface when it is less than about $273 \mathrm{~K}$, and is about $0.88^{3)}$ at $273 \mathrm{~K}$ or $0.9^{15}$ ) at 263 to $253 \mathrm{~K}$. Accordingly, results about the effect of accommodation coefficient cannot be expected from the present experimental data.

\subsection{Apparent density of desublimated solid phase}

The local apparent density of desublimated solid phase is not the same along the axial direction because it depends on the mole fraction of noncondensable gas ${ }^{17}$ and also in the radial direction. However, the total averaged value of apparent density was studied in this work for simplicity in treating the results.

It has been reported by many investigators ${ }^{2,6,20 \text {, }}$ that in most cases the apparent density of desublimated solid phase increases with time due to internal molecular diffusion through the porous parts. Here it was also recognized in the transition region, as shown in Fig. 6. The increase in density is approximately parabolic with time, as in previous experimental results.

The densification rates after about 90 minutes seem to be independent of total pressure and to be slower for a lower temperature of the cooling tube surface. The mean free path of vapor molecules is much larger than the size of porous space at such low pressure as the present experimental one, so that the densification rate is independent of total pressure.

However, the density itself becomes greater for a higher total pressure. This is partly because the generation of crystals and the subsequent growth during the early period will be influenced by the desublimation rate depending on total pressure.

The density was quite low compared with that of the complete crystal, $1.145 \mathrm{~g} / \mathrm{cm}^{3}$ at room temperature $^{8}$, and the aspect was granular and white as shown in Fig. 7. Then the desublimated solid phase of naphthalene will be porous even if there exists little noncondensable gas. 
Similar results were observed in the desublimation of several materials under the continuum region in forced convection by Ueda and Takashima ${ }^{17,187}$. They noted that the apparent density of carbon tetrachloride was almost the same as that of the complete crystal whether noncondensable gas existed or not, but benzene depended on the fraction of noncondensable gas and water also strongly. And the result for $p$-xylene had the same tendency as the present one for naphthalene.

The crystal form is a monoclinic lattice for $p$-xylene and naphthalene, hexagonal for water, rhombic for benzene and cubic for carbon tetrachloride. Accordingly, it may be said that the apparent density and the aspect of desublimated solid matter will be presumed roughly by its crystal form. However, comprehensive relationships between crystal form and operating conditions cannot be confirmed from the few data reported up to present.

\section{2 Local desublimation rate}

Dimensionless local desublimation rate $J^{*}$ is defined by the equation

$$
J^{*}=\frac{\pi\left(r_{2}^{2}-r_{1}^{2}\right)}{G}\left(\frac{j}{2 \pi r_{1}}\right)
$$

where $j[\mathrm{~g} / \mathrm{cm} \cdot \mathrm{s}]$ is the local desublimation rate per unit length of inner cooling tube.

Figure 8 shows the effects of $M a$ on $J^{*}$. The plotted points are the time-averaged values during the quasi-steady period. Their time variations were within the limits of several percent. $Z$ is the dimensionless axial distance normalized by $\Delta r . M a$ is inversely proportional to total pressure, as seen in Eq. (2). The Knudsen number is roughly estimated as 0.1 for Run 12 and 1.1 for Run 13, provided that the mean free path is that of pure naphthalene vapor and the characteristic length is $r_{1}$.

$J^{*}$ decreased exponentially in the axial direction and the gradient became steeper as $M a$ decreased in the transition region. The characteristic was a concave curve in the continuum region but became a convex curve as $M a$ increased. The difference between the results of $J^{*}$ to $Z$ at $M a=0.00836$ (Run 12) and at $M a=0.000963$ (Run 3) was negligibly small, though not illustrated in Fig. 8. Because the mechanism of mass transfer in gaseous phase should be independent of total pressure if the mean free path of vapor molecules is much less than $r_{1}$ or $\Delta r$, the profile of $J^{*}$ should asymptote to a certain curve in the continuum region if the vapor feed rate is kept constant.

\subsection{Reference length of desublimated solid layer}

The shape of desublimated solid layer became longer and thinner with the reduction of total pressure, as shown in Fig. 7.

To treat this difference quantitatively, we define the dimensionless reference length of the solid layer, $Z d^{*}$,

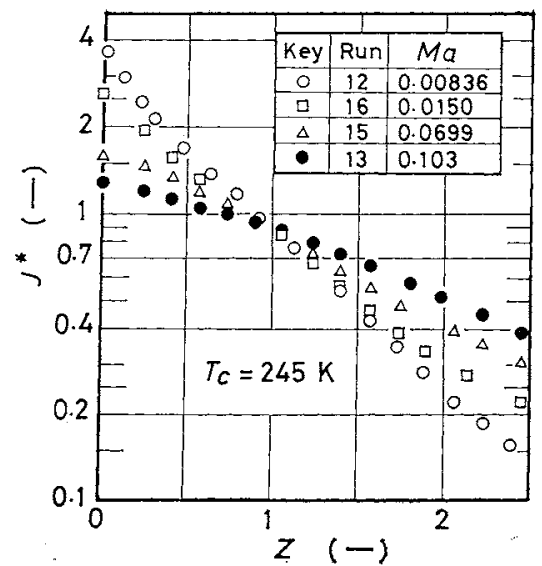

Fig. 8 Effect of $M a$ on local desublimation rate

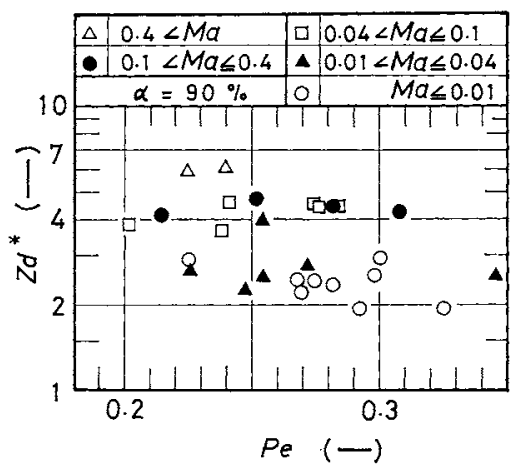

Fig. 9 Effect of $P e$ on $Z d^{*}$

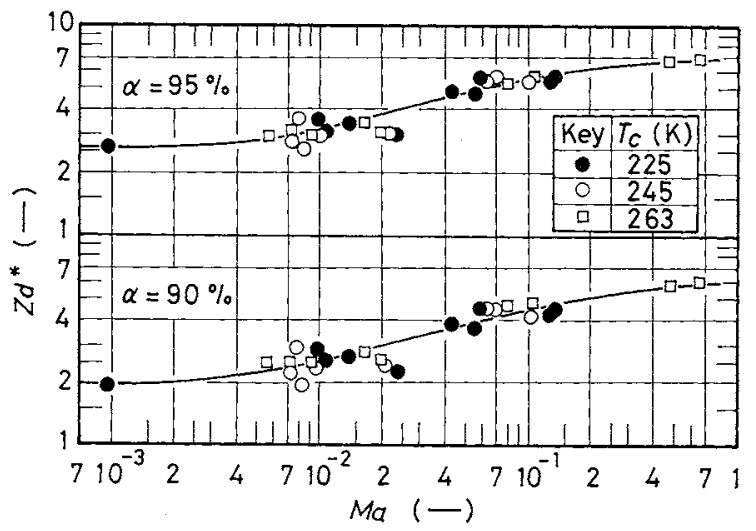

Fig, 10 Effect of $M a$ on $Z d^{*}$

as the length necessary for trapping $\alpha$ percent of the total amount of desublimated matter normalized by $\Delta r$. The value of $Z d^{*}$ when the total feed of vapor becomes just $4 \mathrm{~g}$ is shown in Table 1 at $\alpha=80,90$ and $95 \%$ for each run.

The variation of $Z d^{*}$ to $P e$ was not appreciable, as shown in Fig. 9. If the vapor feed rate is so large that a decrease in total pressure is observable along the axial direction, $Z d^{*}$ should be influenced more or less by $P e$.

Figure 10 shows the variation of $Z d^{*}$ with $M a$. As 
Table 2 Values of $C_{i}^{\prime} s$ in $\ln \left(Z d^{*}\right)=C_{1}+C_{2} \ln (M a)+$ $C_{3}[\ln (M a)]^{2}+C_{4}[\ln (M a)]^{3}$

\begin{tabular}{ccrrr}
$\alpha[\%]$ & \multicolumn{1}{c}{$C_{1}$} & \multicolumn{1}{c}{$C_{2}$} & \multicolumn{1}{c}{$C_{3}$} & \multicolumn{1}{c}{$C_{4}$} \\
\hline 80 & 1.6573 & 0.17194 & -0.03199 & -0.003944 \\
90 & 1.7964 & -0.01636 & -0.08112 & -0.008005 \\
95 & 1.8879 & -0.11726 & -0.10738 & -0.010265 \\
\hline
\end{tabular}

expected, $Z d^{*}$ increased with $M a$ over the range larger than about 0.001 . Because near this point the level of the rarefaction comes to be in the continuum region, $Z d^{*}$ asymptotes to a certain value at $M a$ less than about 0.001 .

The two curved lines in Fig. 10 were obtained by the method of least squares assuming $\ln \left(Z d^{*}\right)=C_{1}+$ $C_{2} \ln (M a)+C_{3}[\ln (M a)]^{2}+C_{4}[\ln (M a)]^{3}$, in which the constants $C_{i}$ 's are shown in Table 2.

Generally, saturated vapor pressure decreases and accommodation coefficient increases if the temperature of the desublimation surface decreases.

If the saturated vapor pressure increases, $Z d^{*}$ becomes larger because the driving force of desublimation is reduced. This dependence is taken into consideration by $M a$.

Because the resistance of phase changing when vapor molecules desublimate or sublimate increases with the reduction of accommodation coefficient, the effect of accommodation coefficient is important in the transition and in the free molecular flow region. As mentioned before, the accommodation coefficient of naphthalene scarcely depends on the temperature of the desublimation surface under the present experimental conditions. Therefore, this effect is not appreciable in Fig. 10.

Within the limits of the present experimental results, it may be said that $Z d^{*}$ depends strongly upon $\mathrm{Ma}$ but scarcely on $\mathrm{Pe}$.

\section{Conclusion}

The following results have been obtained experimentally for desublimation in an annular-tube cold trap operated with a closed system at such low pressures that the Navier-Stokes equations may not be valid.

The desublimation behavior depends strongly upon $M a$ but scarcely upon Pe. (1) The reference length of desublimated solid layer increases with $M a$, where it is larger than 0.001 . (2) The local desublimation rate decreases exponentially along the axial direction and the gradient becomes steeper as $M a$ decreases. The characteristic is a slightly concave curve in the continuum region but becomes a convex curve as $M a$ increases. (3) The apparent density of desublimated solid phase increases parabolically with time. The densification rate becomes slower at lower cooling surface temperature but scarcely depends on total pressure. However, the density itself becomes larger at higher total pressure.

It must be noted that the present results have been obtained for a case limited as follows:

1) There is no effect of the end side of cold trap.

2) The accommodation coefficient of vapor is about 0.9 .

3) The feed rate of vapor is so small that a decrease in total pressure may not be observed along the axial direction.

4) $J^{*}$ and $Z d^{*}$ are obtained from the data during the quasi-steady period after an initial transient period.

$M a$ and $P e$ are new dimensionless parameters given by Eq. (2) and Eq. (3) respectively.

\section{Nomenclature}

$D_{0} \quad=$ binary diffusivity at normal condition $\quad\left[\mathrm{cm}^{2} / \mathrm{s}\right]$

$D_{A B} \quad=$ binary diffusivity $\left[\mathrm{cm}^{2} / \mathrm{s}\right]$

$G \quad=$ vapor feed rate $[\mathrm{g} / \mathrm{hr}]$ or $[\mathrm{g} / \mathrm{s}]$

$J^{*} \quad=$ dimensionless local desublimation rate defined by Eq. (5) [-]

Ma = dimensionless parameter defined by Eq. (2) [-]

$P_{A S} \quad=$ saturated vapor pressure $\quad$ [Pa]

$P_{t} \quad=$ total pressure [Pa]

$\mathrm{Pe}=$ dimensionless parameter defined by Eq. (3) [-]

$r_{1} \quad=$ inner tube radius $\quad[\mathrm{cm}]$

$r_{2} \quad=$ outer tube radius [cm]

$T_{\mathrm{av}} \quad=$ arithmetical average between outer and inner tube surface temperature [K]

$T_{c} \quad=$ temperature of inner cooling tube surface $[\mathrm{K}]$

$t \quad=$ elapsed time [min]

$Z \quad=$ dimensionless axial distance normalized by $A r \quad$ [-]

$Z d^{*} \quad=$ dimensionless reference length of desublimated solid layer normalized by $\Delta r \quad$ [-]

$\alpha$

$=$ percentage of amount of solid matter desublimated on the surface over $Z=0$ to $Z=Z d^{*}$ to total amount

$=$ thickness of desublimated solid layer

$=$ interval between inner and outer tube [cm]

$=$ running time of experiment [min]

$=$ apparent density of desublimated solid phase

$\left[\mathrm{g} / \mathrm{cm}^{3}\right]$

\section{Literature Cited}

1) Biguria, G. and L. A. Wenzel: Ind. Eng. Chem., Fundam., 9, 129 (1970).

2) Brian, P. L. T., R. C. Reid and Y.T. Shah: ibid., 9, 375 (1970).

3) Burrows, G.: J. Appl. Chem., 7, 375 (1957).

4) Dunthorn, D. I. P.: Oak Ridge Report K-L-6220 (1968).

5) Dyer, D. F., R. W. Mayne and J. E. Sunderland: Brit. J. Appl. Phys., 18, 297 (1967).

6) Hayashi, Y., A. Aoki, S. Adachi and K. Hori: Trans. ASME, Series C, J. Heat Transfer, 99, 239 (1977).

7) Hirschfelder, J. O., C. F. Curtiss and R. B. Bird: "Molecular Theory of Gases and Liquids", fourth printing, John Wiley \& Sons, Inc., New York (1967).

8) Katto, Y.: "Dennetsu Gairon", 230, Youkendo, Tokyo (1974).

9) Kawamura, Y.: Dr. Eng. Thesis, Tokyo Institute of 
Technology (1961).

10) Kawamura, Y., H. Makihara and H. Shinagawa: Kagaku Kögaku, 36, 1307 (1972).

11) Mitani, T. and $Y$. Takashima: Preprint of the 13th Autumn Meeting of The Soc. of Chem. Engrs., Japan, at Nagoya p. 684 (1979).

12) Mott-Smith, H. M.: Phys. Review, 82, 885 (1951).

13) Satou, K.: "Bussei Jyousu Suisanho", p. 129, Maruzen, Tokyo (1954).

14) Sherwood, T. K. and C. Johannes: AIChE J., 8, 590 (1962).

15) Toei, R. and M. Okazaki: J. Chem. Eng. Japan, 1, 125 (1968).
16) Toei, R., M. Okazaki and M. Asaeda: ibid., 8, 277 (1975).

17) Ueda, H. and Y. Takashima: ibid., 9, 121 (1976).

18) idem: ibid., 10, 6 (1977).

19) Yamakawa, N. and S. Ohtani: Kagaku Koggaku, 36, 197 (1972).

20) White, J. E. and C. J. Cremers: Paper of AIAA, (74-746)i, 1 (1974).

(A part of this paper was presented at the 6th International Congress of Chemical Engineering, Chemical Equipment Design and Automation, G3.3, Praha, Czechoslovakia, August 21-25, 1978.)

\title{
OVERALL RATE OF OZONE OXIDATION OF CYANIDE IN BUBBLE COLUMN
}

\author{
MASAAKI TERAMOTO, YOSHIHIRO SUGIMOTO, \\ YASUHIRo FUKUI AND HIROSHI TERANISHI \\ Department of Industrial Chemistry, Faculty of Industrial Arts, \\ Kyoto Institute of Technology, Matsugasaki, Kyoto 606
}

\begin{abstract}
Oxidation of cyanide with ozone was carried out using a semi-continuous bubble column and a continuous bubble column. It was found that the overall reaction rate was strongly controlled by liquid-film mass transfer resistance. The observed rate was satisfactorily explained by the theory of gas absorption with chemical reactions if the intrinsic rate expressions of self-decomposition of ozone and the ozone oxidation of cyanide which had been obtained by the present authors were applied to the theoretical equations.

A method for evaluating capacity coefficient of liquid-phase mass transfer from the absorption rate of ozone into water of high $\mathrm{pH}$ is also presented.
\end{abstract}

\section{Introduction}

Ozone is a powerful oxidizing agent second only to fluorine, and it has been recognized that ozone is effective for disinfection, decolorization, deodorization and the destruction of toxic chemicals in wastewater treatment ${ }^{3}$. Another advantage of ozone treatment is that it does not cause secondary pollution, such as the formation of chlorinated compounds encountered in oxidation by chlorine, because of the self-decomposition of ozone in water. However, one of the disadvantages of ozone treatment is that ozone is expensive compared to chlorine. Therefore it is important to apply ozone in decomposing very reactive substances so that it may be effectively utilized.

\footnotetext{
Received July $\overline{25}, 1980$, Correspondence concerning this article should be addressed to M. Teramoto. Y. Sugimoto is now with Prefectural Industrial Research Institute, Nara 630. Y. Fukui is now with Kyoeisha Yushi Chemicals, Nara 630.
}

Cyanide is one of the toxic chemicals which can react with ozone very rapidly, and ozone treatment is a promising method for the treatment of $\mathrm{CN}^{-}$in industrial wastewater. Ozone treatment is, however, carried out in a gas-liquid heterogeneous system where the overall reaction rate as well as the degree of utilization of ozone is influenced by mass transfer resistance, and this complication has made many investigators draw wrong conclusions concerning the reaction kinetics.

The purpose of this paper is to clarify the characteristics of ozone absorption in aqueous cyanide solutions using a semi-continuous bubble column as well as a continuous bubble column, and to explain the experimental data on the basis of the theory of gas absorption with chemical reactions. A method for estimating capacity coefficient of liquid-phase mass transfer of the bubble column from the absorption rate of ozone into water of high $\mathrm{pH}$ is also presented. 